\title{
Electrostrictive behavior of confined water subjected to GPa pressure
}

\author{
Belisa R. H. de Aquino, ${ }^{1}$ H. Ghorbanfekr-Kalashami, ${ }^{1}$ M. Neek-Amal,,${ }^{2,}$ and F. M. Peeters ${ }^{1}$ \\ ${ }^{1}$ Department of Physics, Universiteit Antwerpen, Groenenborgerlaan 171, B-2020 Antwerpen, Belgium \\ ${ }^{2}$ Department of Physics, Shahid Rajaee Teacher Training University, Lavizan, Tehran, Iran
}

(Received 23 October 2017; published 25 April 2018)

\begin{abstract}
Water inside a nanocapillary exhibits unconventional structural and dynamical behavior due to its ordered structure. The confining walls, density, and lateral pressures control profoundly the microscopic structure of trapped water. Here we study the electrostriction of confined water subjected to pressures of the order of GPa for two different setups: (i) a graphene nanochannel containing a constant number of water molecules independent of the height of the channel, (ii) an open nanochannel where water molecules can be exchanged with those in a reservoir. For the former case, a square-rhombic structure of confined water is formed when the height of the channel is $d=6.5 \AA$ having a density of $\rho=1.42 \mathrm{~g} \mathrm{~cm}^{-3}$. By increasing the height of the channel, a transition from a flat to a buckled state occurs, whereas the density rapidly decreases and reaches the bulk density for $d \cong 8.5 \AA$. When a perpendicular electric field is applied, the water structure and the lateral pressure change. For strong electric fields $(\sim 1 \mathrm{~V} / \AA)$, the square-rhombic structure is destroyed. For an open setup, a solid phase of confined water consisting of an imperfect square-rhombic structure is formed. By applying a perpendicular field, the density and phase of confined water change. However, the density and pressure inside the channel decrease as compared to the first setup. Our study is closely related to recent experiments on confined water, and it reveals the sensitivity of the microscopic structure of confined water to the size of the channel, the external electric field, and the experimental setup.
\end{abstract}

DOI: 10.1103/PhysRevB.97.144111

\section{INTRODUCTION}

The microscopic structure of confined water has been studied extensively in the past two decades. Water inside a nanocapillary becomes ordered exhibiting unconventional behavior [1-5]. The confinement size (channel height), the internal pressure, and the microscopic structure of the confining walls are important factors that determine the microscopic structure of confined water [6-12]. The confining walls can weakly interact with water (hydrophobic) or absorb water (hydrophilic), which results in different orientations of the water molecules near the confining walls [13]. Despite extensive theoretical studies on confined water, there are still many unsolved questions, such as the exact orientation of the water molecules over different substrates, which can provide information on the contact angle of the water droplet [14-17]. The density of water, possible phase transitions, and the effects of lateral pressure also are open questions [18-20]. Although there are several reports on the electrostriction of confined water $[10,12,21,22]$, further investigations are needed on the out-of-plane deformations of a single layer of water in the presence of an external perpendicular electric field.

The main challenges, from a theoretical point of view, are (i) the force fields in molecular-dynamics simulations were mostly parametrized using bulk water properties, (ii) the weak interactions (dispersion forces), and (iii) the limiting computational time restricts us to small systems when using density functional theory calculations. However, many of the

*Corresponding author: neekamal@ srttu.edu main properties of confined water can be understood using classical force fields [8,9]. There are several reports on the electrostriction of confined water and the possible electricfield-induced liquid to gas phase transition [12,22]. Classical thermodynamics predicts an increase in the density of bulk liquid when subjected to a weak external field [23], i.e., $\frac{\delta \rho}{\rho} \propto E^{2}$. However, for confined water inside an open channel and subjected to an electric field, evaporation was predicted [22]. This might be due to metastable phases of confined water [22].

Here by using the art of molecular-dynamics (MD) simulations, we study the microscopic structure of a single layer of water confined between two graphene sheets subjected to a pressure of about $1 \mathrm{GPa}$ for two different setups: (i) a graphene nanochannel containing a fixed number of water molecules, and (ii) an open nanochannel that exchanges water molecules with a reservoir via both sides. These setups are relevant for recent experiments [24,25]. We study possible phase transitions and concomitant changes in the density and lateral pressure. Different setups lead to different kinds of structural deformation of confined water. By applying a perpendicular electric field, the dipole moment of the water molecules reorients, resulting in variations in the $\mathrm{H}$-bond network and a structural deformation of confined water. The buckling height varies with the channel height and with the strength and orientation of the external electric field. In our previous work [10], we studied the hysteresis in the polarization of confined water in the presence of an in-plane electric field and found structural transitions that are very different from those reported here (i.e., ordered to disordered phase) for a perpendicular electric field. 


\section{THE MODEL AND THE METHOD}

We employed atomistic molecular-dynamics (MD) simulations implemented in the LAMMPS package [26]. We used the water model TIP4P [27] for water-water interaction. The graphene-water interaction was modeled by the Lennard-Jones (LJ) potential, with $\epsilon_{\mathrm{C}-\mathrm{O}}=0.0949 \mathrm{eV}$ and $\sigma_{\mathrm{C}-\mathrm{O}}=3.28 \AA$. To calculate the lateral $\left(p_{x y}\right)$ and transverse pressure $\left(p_{z}\right)$, we calculated the stress tensor of water molecules inside the channel:

$$
\hat{\sigma}=\left(\begin{array}{lll}
\sigma_{x x} & \sigma_{x y} & \sigma_{x z} \\
\sigma_{y x} & \sigma_{y y} & \sigma_{y z} \\
\sigma_{z x} & \sigma_{z y} & \sigma_{z z}
\end{array}\right),
$$

with $\sigma_{a b}=\sigma_{b a}$ and $a, b=\{x, y, z\}$, i.e., the stress tensor is symmetric, where $\sigma_{a a}\left(\sigma_{a b}\right)$ are the normal (shear) components. The aforementioned pressures can be written as

$$
\begin{gathered}
p_{x y}=-\frac{\left\langle\sigma_{x x}\right\rangle+\left\langle\sigma_{y y}\right\rangle}{2}, \\
p_{z}=-\left\langle\sigma_{z z}\right\rangle,
\end{gathered}
$$

where \langle\rangle denotes the ensemble average. The stress tensor components are calculated by summing the kinetic energy contribution to the energy contribution due to the interactions of each atom, i.e.,

$$
\sigma_{a b}=-\frac{1}{V} \sum_{i=1}^{N}\left[m_{i} v_{i, a} v_{i, b}+r_{i, a} F_{i, b}\right],
$$

where $F_{i, b}$ considers the contribution of pairwise interactions, bonds, and angles constraints, long-range Coulombic interactions required by the TIP4P model, and external applied forces such as the electrical force [28,29]. In Eq. (4), $V=A \bar{d}$ is the effective volume of the channel between two graphene sheets with area $A$ separated by a distance $d$. The effective distance is defined by $\bar{d}=d-\sigma_{\mathrm{C}-\mathrm{O}}$, where $\sigma_{\mathrm{C}-\mathrm{O}}=3.28 \AA$ is a measure of the excluded volume due to the water-wall interaction. More details on pressure calculations of inhomogeneous quasi-twodimensional systems can be found in the Appendix of Ref. [30].

\section{A. Constant number of water molecules: Model A}

First, we simulate a single channel of graphene, which contains a fixed number of water molecules (see Fig. 1). The simulation system consists of a computational unit cell with 840 water molecules confined between two AB-stacked graphene sheets with a total of 5120 carbon atoms and separated by a distance $d$. A top view and two side views of our system for two different channel heights are shown in Figs. 1(a)-1(c). The initial structure of confined water is a square lattice of $\mathrm{O}$ atoms with lattice constant $2.8 \AA$ and a random distribution of $\mathrm{H}$ bonds. Periodic boundary conditions were applied in the $x y$ plane. We set the computational unit cell to be commensurate with the graphene lattice [10]. We performed $N P T$ simulation annealing, using a Nosé-Hoover thermo/barostat to obtain the correct simulation box and interatomic distances. After cooling down the system to nearly zero Kelvin, a square-rhombic ice structure is obtained at a pressure of $0.15 \mathrm{GPa}$. This pressure is in good agreement with previous reports from several groups $[9,10]$. Annealing the system up to nearly
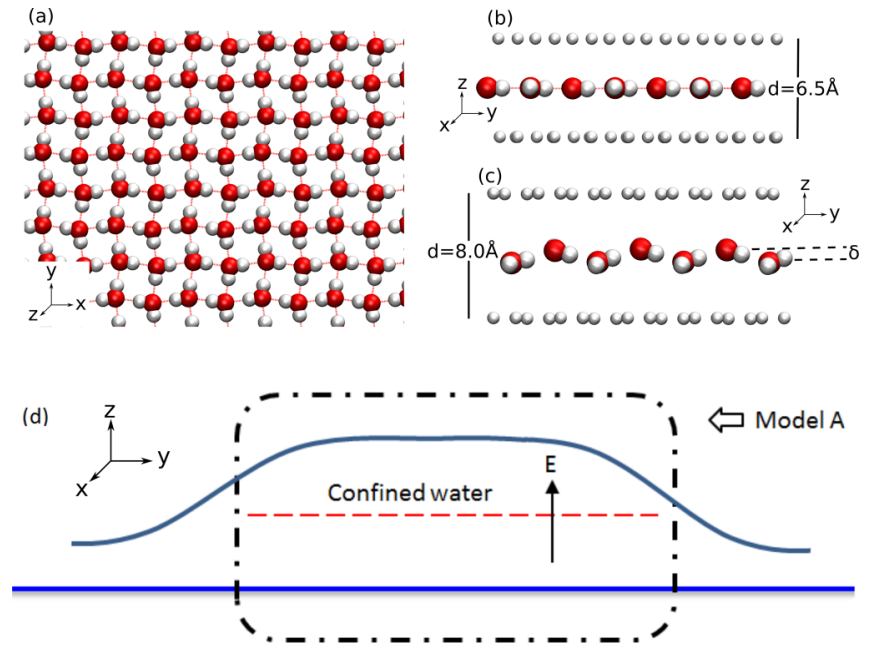

FIG. 1. Model A: Constant number of molecules inside a graphene nanochannel. The square-rhombic lattice for confined water is shown in (a). Red (white) balls are $\mathrm{O}(\mathrm{H})$ atoms of the water molecules. $(\mathrm{b}, \mathrm{c})$ Side view for two different interlayer distances, $d=6.5$ and $8 \AA$, with buckling height $\delta$. (d) A schematic view of the nanoclosure filled by a single layer of water is shown. The dashed rectangle refers to the middle portion of the nanobubble, which is relevant to our model A.

zero Kelvin temperatures allows the system to reach a stable configuration. The obtained structure is in agreement with the minimum-energy configuration obtained in our previous work [10]. Then, an NVT ensemble is adopted using a Nosé-Hoover thermostat in order to preserve the constant volume while an electric field is applied perpendicularly to the graphene sheets. Here, a time-dependent perpendicular electric field is applied in order to obtain the time-dependent evolution of the system. The electric field is varied with a rate of change of $40 \mathrm{~V} \AA^{-1} \mathrm{~ns}^{-1}$ while the system, which is at $T=1 \mathrm{~K}$, stays in thermodynamic equilibrium. A similar procedure was adopted and described in our previous work with an in-plane electric field [10], where rates of the same order of magnitude were used, and the effects of the rate of change on the hysteretic behavior of confined water were investigated. We will show that a very strong perpendicular electric field, in the range $[-3,3] \mathrm{V} / \AA$, will change the microscopic structure of water. Initially, we consider a monolayer of water inside a channel with $d=6.0 \AA$ [9-11]. Subsequently, we increase $d$ in order to elucidate the effects of the channel height on the water layer.

The above system (model A) containing a constant number of molecules is typically realized in graphene bubbles [see Fig. 1(d)] [24]. The number of molecules is constant in the system shown in Figs. 1(b) and 1(c). Our setup corresponds to a perfect nanobubble while in a real nanobubble there are often terraces at the edges of the nanobubble [9] that are not included in our model A. In fact, model A corresponds to the middle portion of the nanobubble, as indicated by the dashed rectangle in Fig. 1(d).

\section{B. Open system: Model B}

A different system (model B) that has been investigated experimentally [25] consists of a nanochannel connected to 


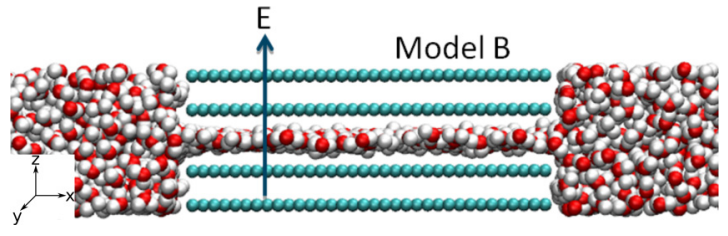

FIG. 2. Model B: An open graphene nanochannel that can exchange water with the reservoir. The electric field is only applied in the channel region.

one reservoir via both sides (in Fig. 2, a channel with $d=$ $7.0 \AA$ is shown). In fact, the channel is made by removing a single layer of graphene from bulk graphite. Recently, Radha et al. successfully fabricated such nanochannels [25]. To model the experimental setup, we considered a graphene nanochannel immersed in a water reservoir in the NPT ensemble, where the temperature, pressure, and subsequently density of water inside the reservoir are controlled by using a Nosé-Hoover thermo/barostat. Here the confined system is open from both sides, and exchange of water molecules with the reservoir is possible. The temperature is kept constant at room temperature. Periodic boundary conditions were applied along the $z(y)$ direction in the reservoir (channel). We found that weaker electric fields (compared to model A) are required to change the microscopic structure of confined water $(\sim 0.5 \mathrm{~V} / \AA)$.

\section{RESULTS: MODEL A}

First we report results for a periodic system that contains a constant number of molecules. This model has recently been widely used in several ab initio studies [31,32]. Here we report the minimum-energy configuration of the confined single layer of water inside the channel subjected to a perpendicular electric field.

\section{A. The microscopic structure: Density and buckling}

Our annealing MD simulations for confined monolayer water between two graphene sheets separated by distances in the range $d=6.0-8.5 \AA$ led to a square-rhombic water monolayer, as depicted in Fig. 1(a) with a flat and buckled structure, for $d=6.5$ and $8.0 \AA$ (buckling height $\delta=1.8 \AA$ ), respectively. The confinement induces structural deformation on the water structure, e.g., by increasing $d$ from $d=6.5$ to $8 \AA$, water molecules spontaneously expand to the larger available space yielding a transition from a flat to a buckled structure. Transitions to buckled phases have been already investigated and reported for similar systems [33]. The transition from the flat to the buckled state by increasing $d$ is identified as two peaks appearing in the density profile of oxygen $(\mathrm{O})$ atoms along the $z$ direction, which are shown in Fig. 3 for $d=6.0,7.25$, and $8.5 \AA$ A In the former case, the $\mathrm{O}$ atoms are narrowly distributed around $z=0$, while in the two latter cases two peaks are located at $z= \pm 0.5$ and $\pm 0.9 \AA$, respectively. The buckling height $\delta$ varies with channel height $d$, and the peaks have a larger variance and larger separation for larger $d$.

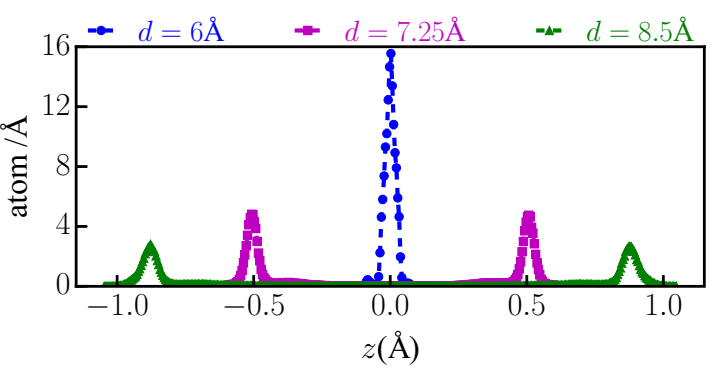

FIG. 3. The density profile of oxygen atoms along the $z$ direction for three different graphene interlayer distances $d$.

The dependence of $\delta$ on $d$ and the water density $\rho$ are shown in Fig. 4. The transition from the flat state $(\delta=0)$ to the buckled state $(\delta \neq 0)$ occurs at $d \approx 6.6 \AA$. For $d \geqslant$ $6.6 \AA$, the buckled structure is determined by the competition between the different interactions in the system, and $\delta$ increases nonlinearly with $d$, reaching $1.75 \AA$ when $d=8.5 \AA$; see Fig. 4 . For $d>8.5 \AA$, the single buckled layer structure no longer exists and a bilayer of confined water is formed. The water density is defined as $\rho=m / v$, where $m$ is the total mass of the water molecules and $v$ is the effective confined volume as defined in Sec. II [19]. As seen from Fig. 4, $\rho$ decreases with $d$ starting from high density $1.42 \mathrm{~g} \mathrm{~cm}^{-3}$ to about $1 \mathrm{~g} \mathrm{~cm}^{-3}$. In fact, the square-rhombic structure corresponds to a high density of water, which can be considered as a new dense phase of ice. Notice that the experimental confirmation of square ice is still controversial [34].

The water structure reported in our study is similar to that reported in Ref. [12], although a denser triangular lattice structure for the confining walls with lattice constant $2.3 \AA$ was used (as compared to our used hexagonal lattice of graphene). Moreover, the pressure values that were reported in Ref. [12] are very different from ours (see below). The buckling heights obtained here (see Fig. 4) are also in line with Ref. [12], e.g., for $d=7.9 \AA$ we obtained $\delta=1.54 \AA$.

\section{B. The effects of a perpendicular electric field}

By applying a perpendicular electric field $E$ (along the $z$ direction), the electric force exerts a torque on the water molecules and causes the dipole moments of the water molecules to align with $E$. Consequently, the square-rhombic lattice structure of the single layer of water is destroyed. Notice

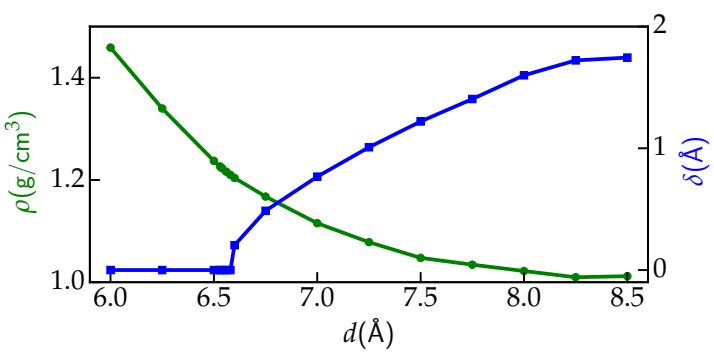

FIG. 4. The variation of buckling height $\delta$ (right scale) and density (left scale) $\rho$ with channel height $d$. For $d=6.6 \AA$, the transition from the flat to the buckled state occurs. 


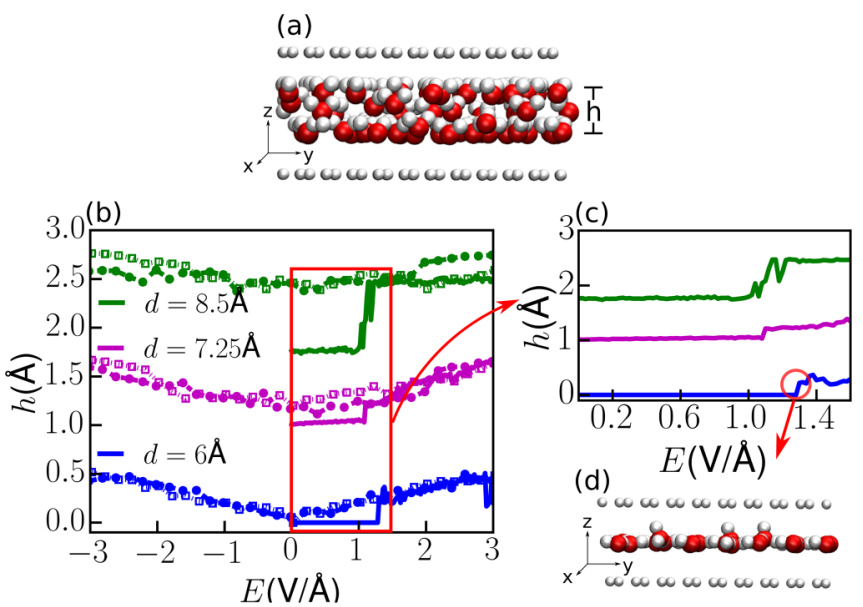

FIG. 5. Side view of confined water for $d=8.5 \AA$, where the shift of $\mathrm{O}$ atoms in the $z$ direction is denoted by $h$. (a) The variation of $h$ with $E$ for different $d$. The solid line corresponds to the increase of $E$ from 0 to $3 \mathrm{~V} / \AA$. The circles (squares) correspond to the decrease (increase) of $E$ from $3(-3)$ to -3 (3) (b). In (c) we show the solid line in the zoomed region. (d) The side view of the water monolayer for $d=6.0 \AA$ indicates that the hydrogen atoms are oriented in the transverse direction when $E=1.3 \mathrm{~V} / \AA$.

that as the initial ordered structure (of $\mathrm{O}$ atoms) is lost, the buckling height $\delta$ is not a well-defined parameter. Therefore, we define the parameter $h$ as the portion of the channel height that is occupied by the $\mathrm{O}$ atoms [see Fig. 5(a)]. For instance, for $d=6 \AA, h=0$ (flat), while for $d=7.25$ and $8.5 \AA$ we found $h=1.0$ and $1.75 \AA$, respectively. The variation of $h$ with an applied electric field is shown in Fig. 5(b) for three different $d$ values. The larger $d$ is, the smaller is the critical electric field $E_{c}$ at which the $\mathrm{H}$ atoms move out of plane, because of the larger degree of freedom of water. Generally, $h$ increases with electric field until it reaches a maximum. By analyzing the maximum value of $h$ for each $d$, we found that the minimum distance between the water molecules and the graphene sheets is about $\sim 2.75 \AA$, which is in agreement with our previous results [8]. By increasing the electric field above a threshold value $\left(E_{c}=1.35 \mathrm{~V} / \AA\right.$ for $\left.d=6 \AA\right)$, the torque exerted by the electric force flips the water molecules, resulting in a sharp increase of $h$ [see Figs. 5(c) and 5(d)]. A sudden change in the water structure due to an increase of electric field was previously reported in Ref. [12].

The alignment of water molecules with an electric field generates a nonzero polarization $Q_{z}$ (i.e., a net dipole along the $z$ direction), which increases more rapidly for larger $d$ values. For weaker fields $\left(E<E_{c}\right)$, the semiflat structure remains unchanged and the total dipole moment varies linearly with $E$. In this quasilinear regime, one can write $Q_{z} \approx \chi E$, where $\chi$ is the polarizability of water. For $d=6.0 \AA(7.25 \AA), \chi \sim 2.38 \times$ $10^{-22} \mathrm{Cnm}^{2} / \mathrm{V}\left(\chi \sim 2.46 \times 10^{-22} \mathrm{Cnm}^{2} / \mathrm{V}\right)$, which is two times larger than the polarizability of bulk water [35]. The reason the polarizability in the bulk is smaller is because the water molecules are surrounded in all directions by other water molecules imposing orientations to establish hydrogen bonds different from the orientation dictated by the external electric field. In the slab confinement, there are only water molecules

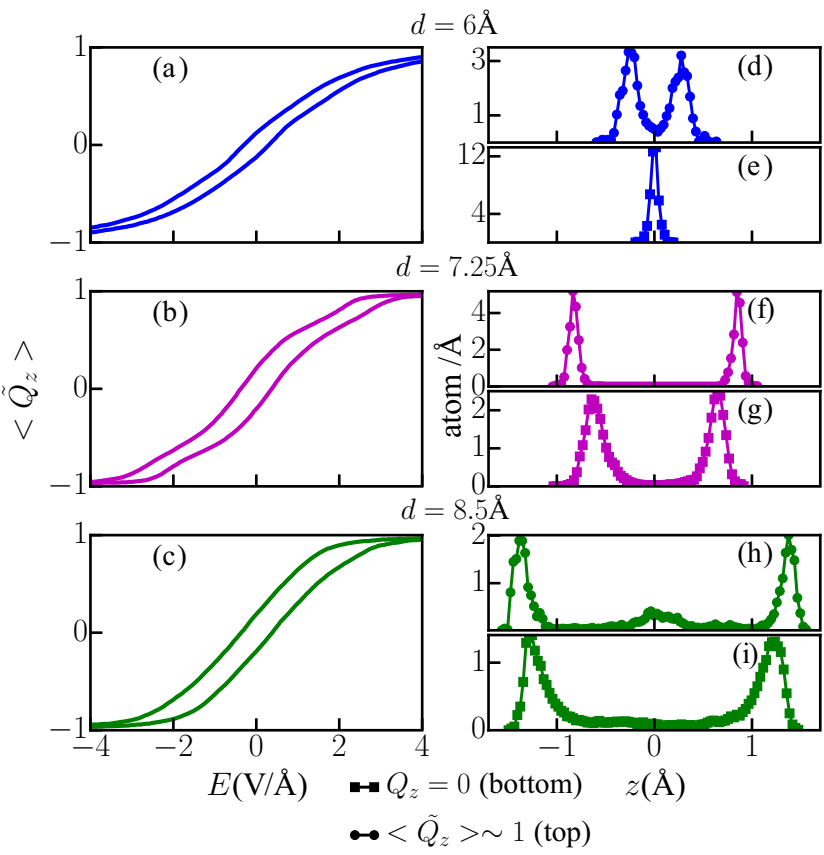

FIG. 6. The variation of the $z$ component of the net dipole moment $\left\langle\tilde{Q}_{z}\right\rangle$ with $E$ for (a) $d=6.0 \AA$, (b) $d=7.25 \AA$, and (c) $d=8.5 \AA$. The corresponding density profiles of $\mathrm{O}$ atoms along the $z$ direction when $\left\langle\tilde{Q}_{z}\right\rangle \sim 1$ (top figures) and when the system recovers $Q_{z}=0$ (bottom figures) are shown in panels (d)-(i).

nearly in the same plane that can oppose the orientation of the electric field [33]. However, for $E>E_{c}$, the water molecules evolve to the out-of-plane space resulting in a deformation of the initial ordered and semiflat structure with an enhancement in the nonlinearity. The variation of $\left\langle\tilde{Q}_{z}\right\rangle$ with $E$ after losing the initial planar structure is shown in Figs. 6(a)-6(c) for $d=6.0,7.25$, and $8.5 \AA$. Here, $\left\langle\tilde{Q}_{z}\right\rangle=\sum_{i}^{N} \frac{Q_{z, i}}{Q_{i}}$, where $N$ is the number of water molecules in the channel, $Q_{i}$ is the dipole moment of the $i$ th molecule, and $Q_{z, i}$ is the component of $Q_{i}$ in the $z$ direction. We found a hysteretic behavior that depends on the graphene interlayer distance $d$. Such hysteresis is different from the previously reported one for an applied in-plane electric field, i.e., in our previous work a larger area under the $Q_{z}$ versus $E$ curve was observed. Also, in a previous work a smaller electric field was needed to reach the maximum dipole moment [10].

We found a significant response of the single layer of water to the applied perpendicular electric field. This can be noticed by comparing the density profiles of the $\mathrm{O}$ atoms along the $z$ axis obtained before applying an electric field, shown in Fig. 3, and Figs. 6(d)-6(i), which show the density profile of $\mathrm{O}$ atoms when $Q_{z}$ is maximum, i.e., $\left\langle\tilde{Q}_{z}\right\rangle \sim 1$ and when the system reaches $Q=0$ again by decreasing $E$. When $d<6.6 \AA$, the initial flat water structure obtained at $E=0$ becomes buckled when $\left\langle\tilde{Q}_{z}\right\rangle \sim 1$. The result for $d=6.0 \AA$ is shown in Fig. 6(d), where the density profile of $\mathrm{O}$ atoms represents two peaks at $z= \pm 0.3 \AA$. The top and side views of the water structure for $d=6.0 \AA$ when $Q_{z}$ is maximum are shown in Fig. 7(a). The top view corresponds to an ordered structure (quasirhombic lattice), while the side view shows 

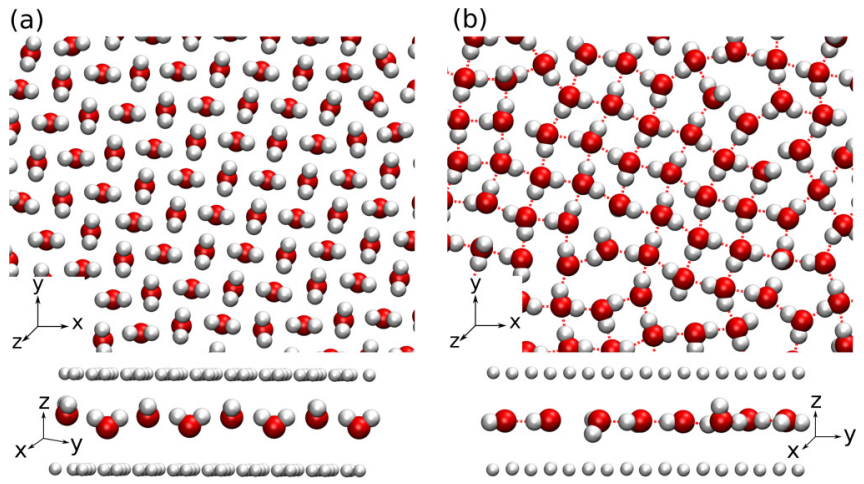

FIG. 7. Top and side views of monolayer confined water for $d=$ $6.0 \AA$ and (a) $\left\langle\tilde{Q}_{z}\right\rangle \sim 1$ and (b) when the system returns to $Q_{z}=0$.

a buckled structure, where neighboring water molecules are perpendicularly oriented with respect to each other. When the system is restored to $Q_{z}=0$, the $\mathrm{O}$ atoms return to the plane occupied before applying $E$ [see Figs. 3 and 6(e)] but the initial square-rhombic structure is no longer found (an amorphous structure appears). The top and side views of the confined water structure for $d=6.0 \AA$ when the system is returned to $Q_{z}=0$ by decreasing $E$ is shown in Fig. 7(b). The top view shows that some defects are found while the $\mathrm{O}$ atoms tend to recover their initial square-rhombic structure corresponding to a larger standard deviation in the density profile of $\mathrm{O}$ atoms. Nonetheless, the side view in Fig. 7(b) shows a nearly flat structure, although there are a few $\mathrm{H}$ atoms parallel to the transverse $z$ direction directed up or down (notice that $\mathrm{O}$ atoms are slightly displaced). When $d \geqslant 6.6 \AA$, the channel height is wide enough to favor the formation of buckled monolayer water, as discussed previously. However, a different buckled structure with a more pronounced buckling is obtained when $Q_{z}$ is maximum. This is shown in Fig. 6(f), where the density profile along the $z$ axis for $d=7.25 \AA$ indicates that the $\mathrm{O}$ atoms are located at $z= \pm 0.85 \AA$ in comparison to the two planes centered at $z= \pm 0.5 \AA$ before $E$ is applied (see Fig. 3). When the electric field is decreased and the system reaches $Q_{z}=0$ again, the $\mathrm{O}$ atoms occupy two planes located at $z= \pm 0.6 \AA$, as shown in Fig. 6(g). In the case of larger $d$, e.g., $d=8.5 \AA$, when $Q_{z}$ is maximum the region between the water planes exhibits a third smaller peak in the density profile of $\mathrm{O}$ atoms centered at $z=0$ [see Fig. 6(h)]. When the system returns to $Q_{z}=0$, the $\mathrm{O}$ atoms are located at $z= \pm 1.1 \AA$ [see Fig. 6(i)] instead of being at $z= \pm 0.9 \AA$ for the initial square-rhombic buckled structure (see Fig. 3).

In Ref. [12], the $\mathrm{O}$ atoms are shifted downward when an electric field is applied. Therefore, the positions of the planes differ slightly from those observed in our simulations (this might be due to including the interaction between the $\mathrm{H}$ atoms and walls in Ref. [12]). However, the water structures and buckling heights reported here for different applied electric fields (and polarizations) are qualitatively in agreement with those of Ref. [12], e.g., a distance of $\sim 2 \AA$ between the two planes occupied by the $\mathrm{O}$ atoms is reported for $d=7.9 \AA$ and $E=0.5 \mathrm{~V} / \AA$, when the reported water structure was similar to the structure shown in Fig. 7(a).

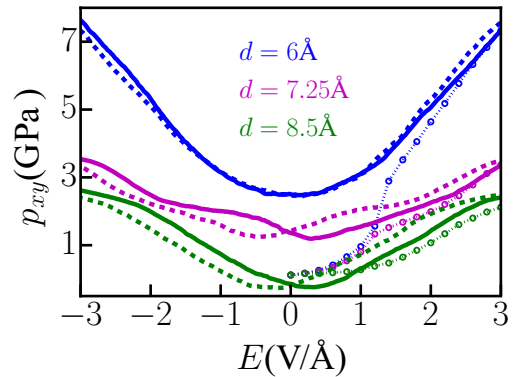

FIG. 8. The variation of the lateral pressure $p_{x y}$ with $E$. The dotted line with circles corresponds to the increase of $E$ from 0 to $3 \mathrm{~V} / \AA$. The dashed (solid) line corresponds to the decrease (increase) of $E$ from $3(-3)$ to -3 (3).

\section{The effects of lateral pressure}

Here we show that the lateral pressure in model A is always of the order of $\mathrm{GPa}$, although in previous work it was reported to be about 1 bar [12]. Figure 8 shows the variation of the lateral pressure $p_{x y}$ with applied $E$. The lateral pressure $p_{x y}$ increases with $E$ until it reaches a maximum value $\left(p_{x y}^{\mathrm{m}}\right)$. The maximum obtained lateral pressure varies significantly with $d$. In fact, we found $p_{x y}^{\mathrm{m}}=12 \mathrm{GPa}$ for $d=6.0 \AA$, while $p_{x y}^{\mathrm{m}}=3.0 \mathrm{GPa}$ for $d=8.5 \AA$. In addition, there is a hysteretic behavior that becomes more prominent when increasing $d$ is observed. The latter can be seen by comparing the solid and dashed curves in Fig. 8 corresponding to an increasing and a decreasing field, respectively. We found that for $d=6 \AA, p_{x y} \propto \alpha E^{2}$, with $\alpha=$ $8.4 \times 10^{-2} \mathrm{C} / \mathrm{m}^{2}$. The nonparabolic shape observed for $d=$ $7.25 \AA$ is related to changes in the water structure.

It is interesting to note that, since the density of the system for a given $d$ is constant by increasing the electric field, $\delta \rho=$ 0 while $\delta p_{\text {tot }} \neq 0$. This is beyond the prediction of classical thermodynamics [23], i.e., $\frac{\delta \rho}{\rho} \propto E^{2}$, and it is due to the effects of confinement. However, in a system in which confined water can exchange molecules with a reservoir, variations in the water density are possible when applying an electric field (see below).

\section{RESULTS: MODEL B}

Hereafter, we turn our attention to model B. We first report the microscopic structure of water inside the channel subjected to a pressure of order $\mathrm{GPa}$, after which the effects of the applied electric field, pressure, and density will be considered.

\section{A. The microscopic structure}

The water structure inside the open channel depends significantly on the pressure in the reservoir and the applied electric field. In Fig. 9, we show different snapshots of confined water inside an open channel where the pressure in the reservoir is kept either at $1 \mathrm{GPa}$ or 1 bar. The confined water at 1 GPa pressure exhibits locally a square-rhombic structure, as observed in model A [see Fig. 1(a)]. Once the electric field is applied, water molecules are squeezed out from the system under ordinary 1 bar pressure, while for the system with GPa pressure only a small decrease in density is observed. The reason for the significant decrease in the density is that the electric field aligns the dipoles of the water molecules in the 


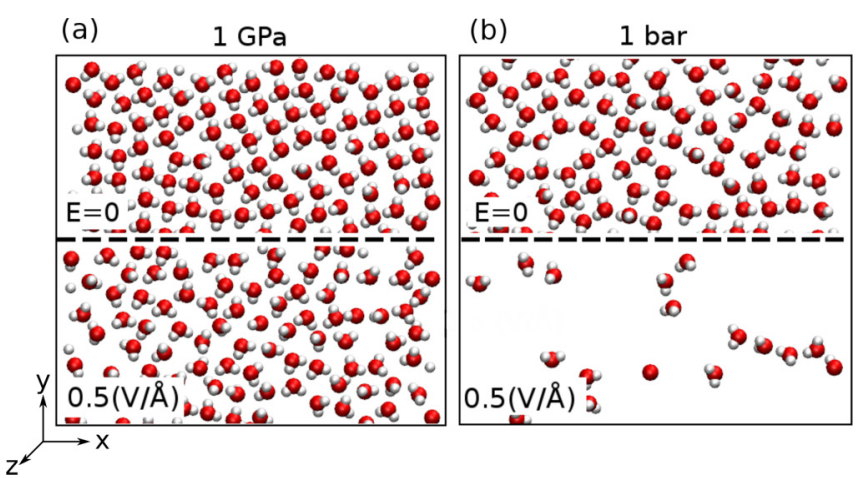

FIG. 9. Four snapshots taken from confined water in the open system where the pressure in the reservoir is kept at 1 bar (a) and $1 \mathrm{GPa}$ (b) and the electric field is 0 (top figures) and $0.5 \mathrm{~V} / \AA$ (bottom figures).

channel, which repel each other. Notice that the electric field is applied only on the water molecules inside the channel and not in the reservoir.

\section{B. The effects of a perpendicular electric field on the total dipole moment and the $\mathrm{H}$ bonds}

We keep the pressure of the reservoir of our open system constant at about 1.0 GPa. After equilibrating the system at room temperature, during $0.1 \mathrm{~ns}$, we started to apply a perpendicular electric field on water in the nanochannel at an average rate of $2 \mathrm{~V}^{-1} \mathrm{~ns}^{-1}$. Figure 10(a) shows the variation of the applied field with time with a maximum of $0.5 \mathrm{~V} / \AA$ after $0.4 \mathrm{~ns}$ of simulation. The corresponding increase in the $z$ component of the net dipole of confined water is shown in Fig. 10(b). We found that when the electric field reaches $0.5 \mathrm{~V} / \AA$, the corresponding dipole moment is $\left\langle\tilde{Q}_{z}\right\rangle \sim 0.25$ $(\sim 0.4)$ for $d=7 \AA(d=10 \AA)$, while in model A the dipole is found to be $\left\langle\tilde{Q}_{z}\right\rangle \sim 0.12$ for $d=(6-8.5) \AA$. Therefore, the field necessary to align the water molecules for this open system setup is smaller than that obtained for model A. Accordingly, the number of $\mathrm{H}$ bonds $\langle H B\rangle$ decreases with increasing electric field; see Fig. 10(c). It is seen that by increasing the electric field, the wider the channel is, the larger is the number of $\mathrm{H}$ bonds per water molecule. Due to confinement effects, the number of $\mathrm{H}$ bonds here is smaller than for bulk water.
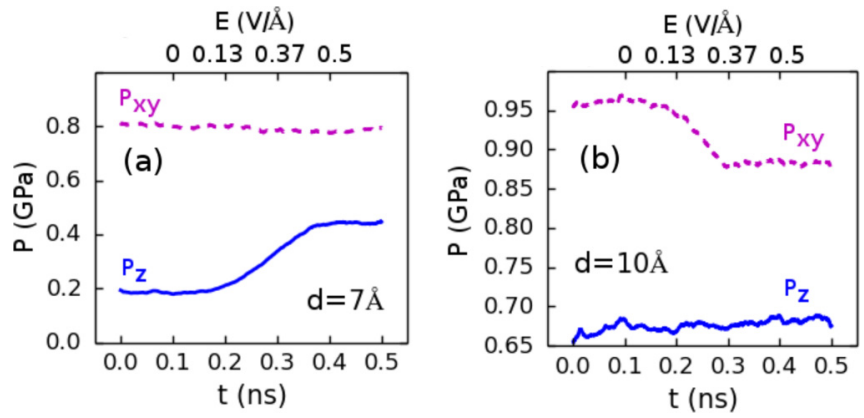

FIG. 11. The variation of pressure of confined water with applied field for two channel heights with $d=7$ and $10 \AA$ (a). Two different components of pressure, i.e., $p_{z}$ and $p_{x y}$, and total pressure inside the channel are shown separately in (b) and (c).

\section{The effects of a perpendicular electric field: Pressure and density}

We found that the influence of the applied electric field on the pressure components inside the nanochannel depends on the channel height. Though the lateral pressure $\left(p_{x y}\right)$ is almost constant in the channel of size $d=7 \AA, p_{z}$ increases significantly with electric field, as shown in Fig. 11(a). However, Fig. 11(b) shows that, for $d=10 \AA, p_{z}$ is nearly constant, which makes the decrease in $p_{x y}$ more significant. The different behaviors of $p_{z}$ with increasing $E$ are due to the smaller available space for water molecules in a channel with height $d=7 \AA$. We conclude that the major role is played by the $p_{z}$ component for narrower channels. The effects of a perpendicular electric field on the pressure of water confined in an open channel are different from the behavior found for the previous setup (see Fig. 8). Moreover, Figs. 11(a) and 11(b) show that after the electric field reaches a maximum of $E=$ $0.5 \mathrm{~V} / \AA$, the pressure components remain nearly constant, which indicates the system is at equilibrium. A similar behavior can be noticed for the net dipole moment $\left\langle\tilde{Q}_{z}\right\rangle$ and the number of $\mathrm{H}$ bonds $\langle H B\rangle$ in Figs. 10(b) and 10(c).

To show possible phase transitions induced by the applied field, we calculated the density variation of confined water (inside the channel) with respect to the applied field. In Fig. 12 we depict the variation of the density with time for two different channels ( $d=7$ and $10 \AA$ ) and two different pressures (1 bar and $1 \mathrm{GPa})$. The results indicate evaporation of water when the pressure of the reservoir is kept at 1 bar. However, when
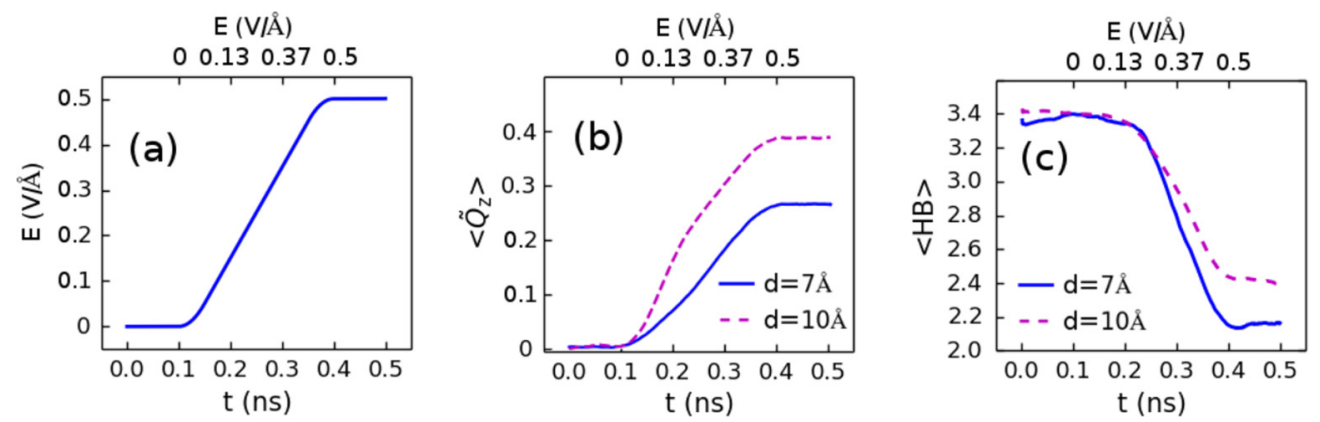

FIG. 10. (a) The applied electric field reaches $0.5 \mathrm{~V} / \AA$ A during $0.4 \mathrm{~ns}$. (b) The variations of the $z$ component of the total dipole moment of confined water with time. (c) The variations in the number of $\mathrm{H}$ bonds with time. 


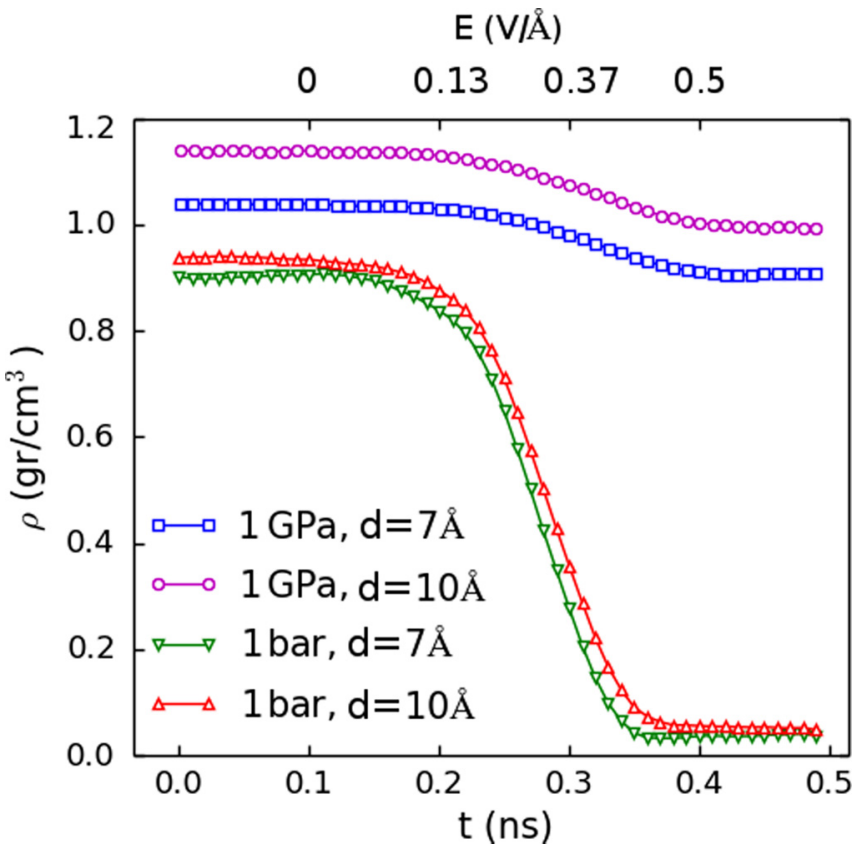

FIG. 12. The variation of density with electric field for nanochannels with height $d=7$ and $10 \AA$ subjected to external pressure 1 bar and $1 \mathrm{GPa}$.

the external pressure is about $1 \mathrm{GPa}$, only a small decrease in density is observed, which indicates a transition from solid to liquid phase. The density decreases from 1.2 to about $0.9 \mathrm{~g} \mathrm{~cm}^{-3}$. Our results for 1 bar are in agreement with those reported by Vaitheeswaran et al. [22]. To confirm the latter effect, the corresponding RDFs were calculated and are shown in Fig. 13(a) for $d=7 \AA$ subjected to GPa pressure and two different electric fields, i.e., 0 and $0.5 \mathrm{~V} / \AA$. The second peak in the RDF disappears when the electric field is $0.5 \mathrm{~V} / \AA$, which is an indication of a transition from the solid to the liquid phase. In Figs. 13(b) and 13(c), we depict the density profiles of $\mathrm{O}$ atoms along the $z$ direction for two systems with $d=7$ and $10 \AA$, respectively. It is shown that for a smaller (larger) channel, a single layer (bilayer) of water is formed. The larger the electric field is, the smaller is the number of water molecules, which is consistent with Fig. 12. The evolution from one to two peaks with increasing $d$ is similar to what we observed for model A, i.e., Fig. 3.

\section{DISCUSSION AND CONCLUDING REMARKS}

In the past few years, several studies [8-12], based on molecular-dynamics simulations, disclosed different physical aspects of confined water between graphene layers. Here, we show that by changing the experimental setup, both the microscopic structure of confined water and the pressure inside the capillary will be different. The pressure, usually called van der Waals (vdW) pressure, is of the order of GPa for channels of height about 6-10 $\AA$ [9]. Notice that in a real experimental situation, the $\mathrm{vdW}$ forces between the two graphene sheets are attractive, which minimizes the contact region below the graphene sheet of a nanobubble. If water is trapped between the sheets, the bubble will shrink in size until the elastic energy of the graphene sheet and the adhesion between the substrate are balanced, i.e., a stable nanobubble is formed [36,37]. This motivated us to introduce model A. If enough water molecules are trapped in this nanobubble, a single layer of water molecules can be formed, which is under the influence of large lateral and vertical pressures. For such a system, the number of water molecules is constant, the temperature is kept nearly at zero Kelvin, and the height of the graphene nanochannel determines the water structure either to be flat or buckled. The structure of a monolayer of water for $d \leqslant 8.5 \AA$ was found to be square-rhombic. We found that in a nanochannel with a constant number of water molecules, the buckling height $\delta$ increases with the height of the channel; it reaches $\sim 1.75 \AA$ for a channel of $d \approx 8.5 \AA$. On the other hand, the three-dimensional water density $\rho$ decreases with increasing $d$.

Moreover, we studied the response of the confined monolayer of water to an out-of-plane electric field. By applying an external electric field, the lateral pressure varies significantly and becomes larger than $1 \mathrm{GPa}$. The smaller $d$ is, the larger is the lateral pressure. The net dipole moment of confined water increases linearly with electric field for weak fields and saturates at large field. For strong electric fields, the squarerhombic structure is lost and the net dipole moment exhibits a hysteretic behavior. The minimum value of the electric field that is needed to deform the square-rhombic structure was found to be about $1.3 \mathrm{~V} / \AA$ for $d=8.5 \AA$. Therefore, we conclude that the confined monolayer of water in the first setup is very stable and resists against electric fields of about $<1 \mathrm{~V} / \AA$ Å. The very strong electric field applied on the confined water may ionize the water molecules in real situations, and therefore it may appear that our conclusions in the case of the
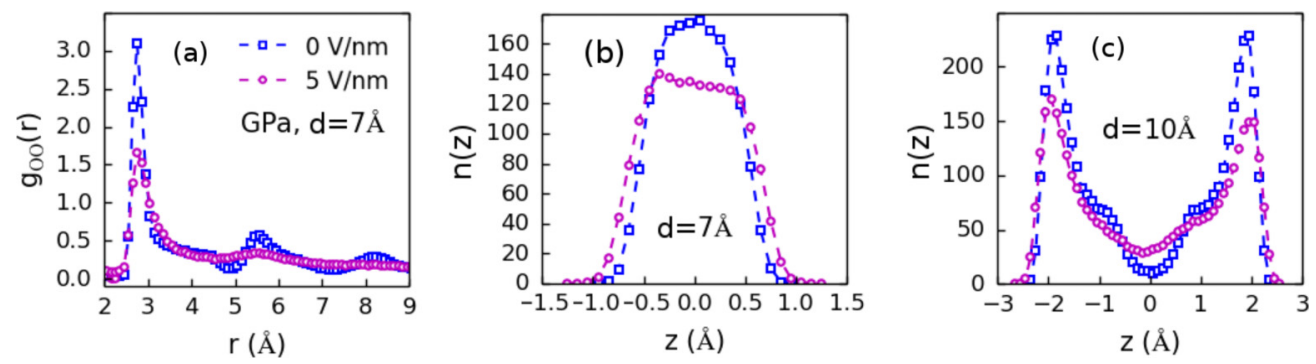

FIG. 13. The radial distribution function for a system with $d=7 \AA$ (a) and the corresponding density profiles along the $z$ direction for $d=7 \AA$ (b) and $d=10 \AA$ (c). The results for two different electric fields are compared. 
first simulated setup have some limitations. However, although bulk water is ionized under such a strong electric field [38], in recent $a b$ initio calculations on confined water it was found that ionization of square-rhombic ice did not occur at such high electric fields, and therefore we believe that our results are meaningful $[31,32]$.

In the second part of this study, we reported the results for an open channel where water molecules can be exchanged with a reservoir via both sides at $T=300 \mathrm{~K}$. Much weaker electric fields (as compared to the first setup) are required to induce deformation in the water structure. By controlling the pressure of the reservoir, we were able to control the pressure inside the channel. However, we found that the previously found squarerhombic structure now contains defects. The transition from solid to gas phase (albeit very few water molecules remain in the channel) due to the applied field occurs only when ambient pressures are applied on the reservoir, i.e., 1 bar. However, keeping GPa pressure at the reservoir, we observed a transition from solid to liquid phase.

Our study demonstrated the sensitivity of the microscopic structure of a confined single layer of water to different geometries of the simulation setup and external parameters.

\section{ACKNOWLEDGMENTS}

This work was supported by the Fund for Scientific Research-Flanders (FWO-Vl) and the Methusalem programe.
[1] M. Majumder, N. Chopra, R. Andrews, and B. J. Hinds, Nature (London) 438, 44 (2005).

[2] J. K. Holt, H. G. Park, Y. Wang, M. Stadermann, A. B. Artyukhin, C. P. Grigoropoulos, A. Noy, and O. Bakajin, Science 312, 1034 (2006).

[3] J. K. Holt, Adv. Mater. 21, 3542 (2009).

[4] X. Qin, Q. Yuan, Y. Zhao, S. Xie, and Z. Liu, Nano Lett. 11, 2173 (2011).

[5] J. Köfinger, G. Hummer, and C. Dellago, Proc. Natl. Acad. Sci. USA 105, 13218 (2008).

[6] R. Zangi and A. E. Mark, Phys. Rev. Lett. 91, 025502 (2003).

[7] R. Zangi and A. E. Mark, J. Chem. Phys. 119, 1964 (2003).

[8] M. Neek-Amal, F. M. Peeters, I. V. Grigorieva, and A. K. Geim, ACS Nano 10, 3685 (2016).

[9] G. Algara-Siller, O. Lehtinen, F. C. Wang, R. R. Nair, U. Kaiser, H. A. Wu, A. K. Geim, and I. V. Grigorieva, Nature (London) 519, 443 (2015).

[10] M. Sobrino Fernandez, F. M. Peeters, and M. Neek-Amal, Phys. Rev. B 94, 045436 (2016).

[11] Y. Zhu, F. Wang, J. Bai, X. C. Zeng, and H. Wu, ACS Nano 9, 12197 (2015).

[12] H. Qiu and W. Guo, Phys. Rev. Lett. 110, 195701 (2013).

[13] G. D. Bixler and B. Bhushan, Soft Matter 9, 1620 (2013).

[14] X. Li, H. Qiu, X. Liu, J. Yin, and W. Guo, Adv. Funct. Mater. 27, 1603181 (2017).

[15] H. Li and X. C. Zeng, ACS Nano 6, 2401 (2012).

[16] F. Sedlmeier, J. Janecek, C. Sendner, L. Bocquet, R. R. Netz, and D. Horinek, Biointerphases 3, FC23 (2008).

[17] M. Sobrino Fernandez Mario, M. Neek-Amal, and F. M. Peeters, Phys. Rev. B 92, 245428 (2015).

[18] V. Satarifard, M. Mousaei, F. Hadadi, J. Dix, M. Sobrino Fernandez, P. Carbone, J. Beheshtian, F. M. Peeters, and M. Neek-Amal, Phys. Rev. B 95, 064105 (2017).

[19] P. Kumar, S. V. Buldyrev, F. W. Starr, N. Giovambattista, and H. E. Stanley, Phys. Rev. E 72, 051503 (2005).

[20] H. Mosaddeghi, S. Alavi, M. H. Kowsari, and B. Najafi, J. Chem. Phys. 137, 184703 (2012).
[21] R. Zangi and A. E. Mark, J. Chem. Phys. 120, 7123 (2004).

[22] S. Vaitheeswaran, H. Yin, and J. C. Rasaiah, J. Phys. Chem. B 109, 6629 (2005).

[23] H. S. Frank, J. Chem. Phys. 23, 2023 (1955).

[24] K. S. Vasu, E. Prestat, J. Abraham, J. Dix, R. J. Kashtiban, J. Beheshtian, J. Sloan, P. Carbone, M. Neek-Amal, S. J. Haigh, A. K. Geim, and R. R. Nair, Nat. Commun. 7, 12168 (2016).

[25] B. Radha, A. Esfandiar, F. C. Wang, A. P. Rooney, K. Gopinadhan, A. Keerthi, A. Mishchenko, A. Janardanan, P. Blake, L. Fumagalli, M. Lozada-Hidalgo, S. Garaj, S. J. Haigh, I. V. Grigorieva, H. A. Wu, and A. K. Geim, Nature (London) 538, 222 (2016).

[26] S. Plimpton, J. Comput. Phys. 117, 1 (1995).

[27] W. L. Jorgensen, J. Chandrasekhar, J. D. Madura, R. W. Impey, and M. L. Klein, J. Chem. Phys. 79, 926 (1983).

[28] A. P. Thompson, S. J. Plimpton, and W. Mattson, J. Chem. Phys. 131, 154107 (2009).

[29] T. W. Sirk, S. Moore, and E. F. Brown, J. Chem. Phys. 138, 064505 (2013).

[30] R. Zangi and S. A. Rice, Phys. Rev. E 58, 7529 (1998).

[31] F. Corsetti, J. Zubeltzu, and E. Artacho, Phys. Rev. Lett. 116, 085901 (2016); F. Corsetti, P. Matthews, and E. Artacho, Sci. Rep. 6, 18651 (2016).

[32] J. Chen, G. Schusteritsch, C. J. Pickard, C. G. Salzmann, and A. Michaelides, Phys. Rev. Lett. 116, 025501 (2016).

[33] R. Zangi, J. Phys.: Condens. Matter 16, S5371 (2004).

[34] W. Zhou, K. Yin, C. Wang, Y. Zhang, T. Xu, A. Borisevich, L. Sun, J. C. Idrobo, M. F. Chisholm, S. T. Pantelides, R. F. Klie, and A. R. Lupini, Nature (London) 528, 7583 (2015).

[35] I. V. Leontyev and A. A. Stuchebrukhov, J. Chem. Theor. Comput. 6, 3153 (2010).

[36] H. Ghorbanfekr-Kalashami, K. S. Vasu, R. R. Nair, F. M. Peeters, and M. Neek-Amal, Nat. Commun. 8, 15844 (2017).

[37] K. Yue, W. Gao, R. Huang, and K. M. Liechti, J. Appl. Phys. 112, 083512 (2012).

[38] M. Chaplin, (n.d.). Water Structure and Science. Retrieved from http://www1.1sbu.ac.uk/water/. 